\begin{tabular}{|c|c|}
\hline Title & $\begin{array}{l}\text { Excitation energy dependence of initial phase shift in Kerr rotation of resident electron spin polarization in a CdTe } \\
\text { single quantum well }\end{array}$ \\
\hline Author(s) & Y an, L. -P.; Takamure, T.; Kaji, R.; Karczewski, G.; Takeyama, S.; A dachi, S. \\
\hline Citation & $\begin{array}{l}\text { Phy sica status solidi B-basic solid state phy sics, 254(2), } 1600449 \\
\text { https://doi.org/10.1002/pssb.201600449 }\end{array}$ \\
\hline Issue Date & $2017-02$ \\
\hline Doc URL & http:/hdl.handle.net/2115/68241 \\
\hline Rights & $\begin{array}{l}\text { This is the peer reviewed version of the following article: [physica status solidi (b) V olume } 254, \text { I ssue } 2 \\
\text { February } 2017 \\
1600449 \text {, which has been published in final form at } \\
\text { http://onlinelibrary.wiley.com/doi } / 10.1002 / p s s b .201600449 / \text { abstract. This article may be used for non-commercial } \\
\text { purposes in accordance with Wiley Terms and Conditions for Self-A rchiving. }\end{array}$ \\
\hline Type & article (author version) \\
\hline File Information & Y an_pssb_rev.pdf \\
\hline
\end{tabular}

Instructions for use 


\title{
Excitation energy dependence of initial phase shift in Kerr rotation of resident electron spin polarization in a CdTe single quantum well
}

\author{
L.-P. Yan ${ }^{1,2}$ T. Takamure ${ }^{1}$, R. Kaji ${ }^{1}$, G. Karczewski ${ }^{3}$, S. Takeyama ${ }^{4}$, S. Adachi ${ }^{*}, 1$ \\ ${ }^{1}$ Department of Applied Physics, Hokkaido University, Sapporo 060-8628, Japan \\ ${ }^{2}$ Research Center of Advanced Materials Science and Technology, Taiyuan University of Technology, Taiyuan 030024, PR China \\ ${ }^{3}$ Institute of Physics, Polish Academy of Sciences, 02-668 Warsaw, Poland \\ ${ }^{4}$ Institute for Solid State Physics, University of Tokyo, 277-8581 Kashiwa, Japan
}

Received XXXX, revised XXXX, accepted XXXX

Published online XXXX

Key words: spin precession, quantum well, CdTe, time-resolved Kerr rotation

${ }^{*}$ Corresponding author: e-mail adachi-s@eng.hokudai.ac.jp, Phone: +81-11-7066709, Fax: +81-11-7066709

The generation dynamics of a resident electron spin polarization involve the formation and transformation of the associated optically excited states depending on the excitation photon energy. Initial phase shift in the precession of a resident electron spin polarization gives the important clues to reveal the interplay between the associated excited states and resident electrons. In this work, the excitation energy dependence of the initial phase shift in Kerr rotation of a resident electron spin polarization is investigated in a $\mathrm{CdTe} / \mathrm{Cd}_{0.85} \mathrm{Mg}_{0.15} \mathrm{Te}$ single quantum well under a low magnetic field. Through the careful analysis of the spin precession, the excited electron heavy-hole pairs show the unique spin dynamics when the excitation energy is increasing past the neutral exciton resonance. Meanwhile, the negative initial phase shift becomes significantly large in magnitude. The larger initial phase shift and the unique precession traces are caused by the transformation from neutral excitons to negative trions with accompanying the hole spin-flip. The exciton-to-trion transformation time is estimated experimentally to be $\sim 160$ ps.
1 Introduction Resident electron spin polarization (RESP) in semiconductor nanostructures has attracted considerable attention as one of key components in potential application of spin degree of freedom to electro-optic devices and quantum information processing [1-4] as well as in fundamental academic studies. Compared to the charge based devices, the spin-based electronics or the semiconductor spintronics have the potential to yield new device functionalities, to overcome the shortcoming of heating and power consumption, and to improve the performances with non-volatility and the increased data processing speed in future applications. These applications could benefit from a long spin coherence of the RESP. Because the RESP can exist after the recombination of the associated optically excited states such as negatively charged excitons (trions) and the spin coherence is not limited by the fast recombination process.

Importantly, the initialization of the RESP by resonant absorption of circularly polarized pulses is essentially dictated by the instantaneous formation of a trion state $(T)$, which is a negatively charged exciton which consists of two electrons with the paired spins (singlet state) and one hole [5]. In the past several years, the important role played by trions for the properties of RESP in the quantum wells (QWs) has been highlighted [6,7]. It is also important to have the correct scenario of the role of neutral excitons $\left(X^{0}\right)$, which allows for better control and study of the trion spin dynamics and RESP formation [5,8]. However, rather few groups have investigated the action of the photocreated electron-heavy holes $(e-h h)$ pairs on RESP in QWs 
systematically. The effects of the excited $e-h h$ pairs are easy to be neglected due to their short lifetime [9]. However, we cannot rule out the potential influence of $e$ - $h h$ pairs on RESP, even though the exciton formation and transformation to trion make the RESP dynamics rather complicated. We have observed and analyzed the initial phase shift (IPS) phenomenon of the time-resolved Kerr rotation (TRKR) signals of the RESP component at time origin $(t=0)$, as shown later (Fig. 1) [10]. Since the IPS gives the important clues concerning the generation processes of RESP, we consider that the experimental and theoretical studies of IPS play a crucial role in the study of RESP.

In this work, we investigate mainly the distinct negative IPS in a temporal evolution of the RESP with different photon energy in a CdTe single QW (SQW) using a TRKR technique. CdTe consists of the isotopes with $I_{\mathrm{Cd}}=1 / 2$, $p_{\mathrm{Cd}}=25 \%, I_{\mathrm{Te}}=1 / 2, p_{\mathrm{Te}}=8 \%$ and the other isotopes with $I_{\mathrm{Cd}, \mathrm{Te}}=0$, where $I_{i}, p_{i}$ are nuclear spin and natural abundance of the isotope $i$, respectively. Therefore, $\mathrm{CdTe}$ is one of the attractive compounds in terms of smaller effects of hyperfine interaction and quadrupolar interaction induced by strain due to small abundance of the isotopes with onehalf nuclear spin, compared to III-V semiconductors such as GaAs and InAs [11].

Both the exciton spin relaxation and the transformation from $X^{0}$ to $T$ have been taken into account in our model, and the transformation time could be deduced from the measurements. Through the analysis and interpretation of RESP temporal evolutions, the contribution of the $e-h h$ pairs to RESP dynamics has been estimated under a low applied magnetic field.

2 Experimental procedure The sample used in this study was a nominally undoped $\mathrm{CdTe} / \mathrm{Cd}_{0.85} \mathrm{Mg}_{0.15} \mathrm{Te}$ SQW with a well width of $100 \AA$ grown by molecularbeam epitaxy on a (100)-oriented GaAs substrate. The resident electrons are supposed to be localized in the well with a density of $n_{e} \sim 2 \times 10^{10} \mathrm{~cm}^{-2}$, which was estimated from the relation between $X^{0}$ and $T$ oscillator strength in the reflectivity spectra [12]. The resident electrons can interact with optically injected $e-h h$ pairs to form negative trion states. The n-type doping and the formation of negative trions were confirmed by TRKR measurements [10, 13].

Figure 1 shows the photoluminescence (PL) spectra measured under a continuous excitation with a photon energy of $1.650 \mathrm{eV}$ at $10 \mathrm{~K}$. The PL spectra can be fitted well by the sum of two Lorentz functions, corresponding to the negatively charged trion resonant transition $T$ (at the low energy peak: $1.6110 \mathrm{eV}$ ) and the neutral exciton resonant transition $X^{0}$ (at the high energy peak: $1.6133 \mathrm{eV}$ ). Those are clearly separated by the trion binding energy of $2.3 \mathrm{meV}$, which is beneficial to the selective excitation of trions or excitons. The trions are generated through the lowest-energy optical transition in the system. From the in-

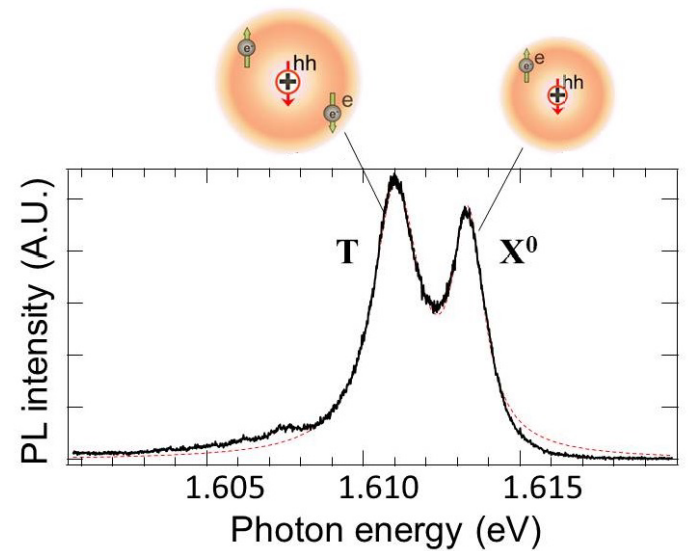

Figure 1 (Color Online) PL spectra of the studied $\mathrm{CdTe} / \mathrm{Cd}_{0.85} \mathrm{Mg}_{0.15} \mathrm{Te}$ SQW measured under continuous optical excitation with the photon energy of $1.650 \mathrm{eV}$ at 10 K. Negative trions $(T)$ and neutral excitons $\left(X^{0}\right)$ are clearly separated by a trion binding energy of $2.3 \mathrm{meV}$. The dashed line indicates a fitting by Lorentzian shaped spectra. The upper illustrations are the examples of $T$ and $X^{0}$.

dependent time-resolved PL measurements using a streak scope, the recombination times of $T$ and $X^{0}$ were found to be relatively short ( $\sim 85$ ps and $\sim 89 \mathrm{ps}$, respectively).

In this experimental report, the dynamical evolution of the created RESP and the interplay with trions and excitons were investigated mainly via the excitation energy dependence using the TRKR technique. A circularly polarized pump pulse was incident along the QW growth direction $(z)$ and the created carrier spin polarization was monitored. A magnetic field was applied in the $x$-direction (Voigt geometry). In this case, the spin polarizations were prepared orthogonal to the applied field and started to precess with Larmor angular frequency $g_{\perp} \mu_{\mathrm{B}} B_{x} / \hbar$ in the $y$ - $z$ plane immediately after optical initialization. Here, $g_{\perp}$ is an inplane electron $g$-factor and $\mu_{\mathrm{B}}$ is a Bohr magneton $(\sim 58$ $\mu \mathrm{eV} / \mathrm{T}$ ). The value for $g_{\perp}=-1.468 \pm 0.003$ of this sample was found in our previous study [11]. Since the pump and probe beams are in the $x-z$ plane that is orthogonal to the spin precession plane ( $y-z$ plane), any extrinsic initial phase shifts of the electron spin precession, for instance, due to an oblique incidence of the pump pulse [14], do not arise.

Both pump and probe pulses were supplied by a modelocked Ti: sapphire oscillator with a pulse width of $\sim 2$ ps and a repetition rate of $76 \mathrm{MHz}$. The spectral width of the pump and probe pulses $(<1 \mathrm{meV})$ was narrower compared to the trion and exciton spectra as shown in Fig. 1. Similar to a standard TRKR setup, the linearly polarized probe pulse with a controlled delay time samples the carrier spin polarization in the central area excited by a pump pulse. A Kerr rotation of the probe polarization was measured sensitively by using a polarization bridge and double lock-in 


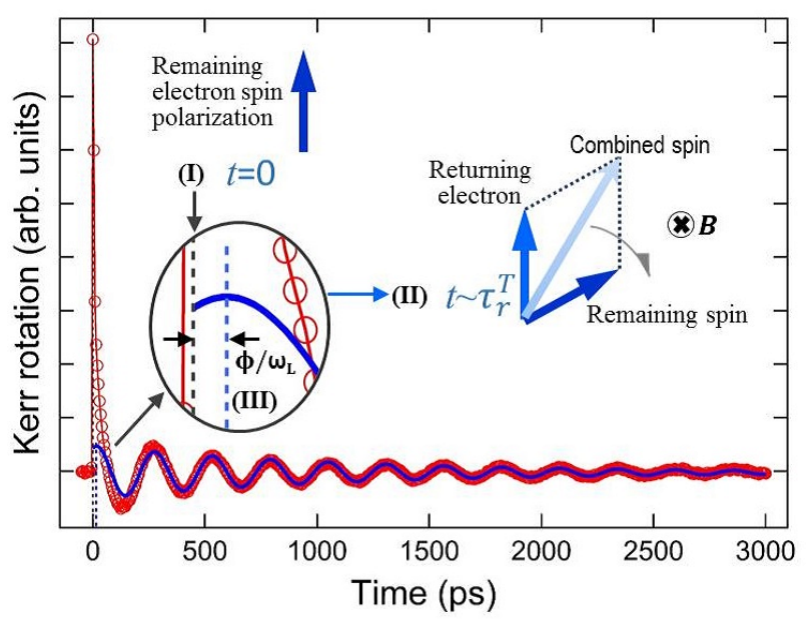

Figure 2 (Color Online) A TRKR signal (open circles) and its fitting curve of the RESP precession (solid line) in the case of the trion resonant excitation ( $189 \mathrm{mT}$ and $10 \mathrm{~K}$ ). Insets show the IPS generation processes: (I) The SQW is polarized with remaining $+1 / 2$ electrons by a $\sigma^{-}$excitation; (II) After the trion recombination accompanying by hole-spin flip, the returning $+1 / 2$ electron contributes a combined RESP, resulting a phase-shift corresponding to the initial spin polarization; (III) The negative IPS $\phi$ of RESP is shown by a backward temporal shift $\phi / \omega_{\mathrm{L}}=-19.6 \mathrm{ps}$.

detection technique. Based on a reported diffusion constant of $T$ and $X^{0}\left(D \sim 10 \mathrm{~cm}^{2} / \mathrm{s}\right)$ at $10 \mathrm{~K}$ [15], we assumed reasonably that the excited carriers could not escape out of a probed spot ( $\sim 100-\mu$ m diameter) during the observed time range $(\sim 3 \mathrm{~ns})$. Therefore, the electron diffusion was not taken into account in this study.

The probe power was fixed at $0.3 \mathrm{~mW}$ and the pump power closes to $8 \mathrm{~mW}$. The QW sample was mounted in an optical cryostat and the temperature was kept at $\sim 10 \mathrm{~K}$. In order to detect the initial phase of RESP precession clearly, we used a relatively low magnetic field $(<200 \mathrm{mT})$.

\section{Results and discussion}

3.1 Initial phase shift Here, we explain the IPS of the spin precession briefly for the trion resonant excitation case at the beginning. The narrower spectral pulse compared to the trion spectrum and the large binding energy allow the excitation of the negative trions selectively. Then, under a $\sigma^{-}$pulsed excitation tuned at $769.7 \mathrm{~nm}$ (trion resonance), a typical TRKR signal and the fitting curve of the ensemble spin precession are obtained at $B_{x}=189 \mathrm{mT}$ and $10 \mathrm{~K}$ as shown in Fig. 2. In the case of the trion resonant excitation, the RESP generation process is relatively simple as follows; the $\sigma^{-}$polarized pulse at $t=0$ can generate the $e-h h$ pairs $(\uparrow \Downarrow)$ selectively ((I) in Fig. 2$)$, where $\uparrow(\Downarrow)$ indicates the up-spin electrons (down-spin holes). Each $e-h h$ pair captures one electron with opposite spin $(\downarrow)$ from the resident electron ensemble and forms a negative trion $(\uparrow \downarrow \Downarrow)$ with an angular momentum of $-3 / 2$. At this moment, the resident electron ensemble loses the spin balance. Meanwhile, a nonzero net RESP comes out along the light propagation direction. After the trion recombination (the recombination time $\tau_{\mathrm{r}}^{T} \sim 85 \mathrm{ps}$ ), the remaining electrons return to the resident electron ensemble and can change RESP ((II) in Fig. 2). Depending on the hole spin-flip time, the trion lifetime, and the precession period (Larmor frequency), the amplitude and phase of the TRKR signals originated from RESP may change intricately [10]. Since the lifetime of the trions is less than $100 \mathrm{ps}$, the oscillatory dumping component with a long coherence in the TRKR signal can confidently be associated with RESP. Note that the negative trions indicate a simple decay due to the short lifetime without a spin-precession since the total in-plane $\mathrm{g}$-factor of the negative trion is zero in a QW. Also, since the pulse width $(\sim 2 \mathrm{ps})$ is much shorter than the precession period of RESP, the spin rotation during the pulse can be neglected. The generation process in the case of trion resonant excitation and the first observation of the negative IPS are detailed in the previous study [10].

Under the existence of excitons, the electrons of excitons should affect the TRKR signals. As a result, a typical experimental TRKR signal $S_{z}(t)$ can be described generally by the following function:

$$
\begin{aligned}
S_{z}(t) & =A^{T} \exp \left(-t / \tau^{T}\right)+A^{X, h} \exp \left(-t / \tau^{X, h}\right) \\
& +A^{X, e} \exp \left(-t / \tau^{X, e}\right) \cos \left(\omega_{\mathrm{L}} t+\phi_{0}\right) \\
& +A^{e} \exp \left(-t / \tau_{\mathrm{s}}^{e}\right) \cos \left(\omega_{\mathrm{L}} t+\phi\right)
\end{aligned}
$$

where the coefficients $A^{T}, A^{X, e(h)}$, and $A^{e}$ are the amplitudes of trions' holes, excitons' electrons (holes), and $\operatorname{RESP} ; \tau^{T}, \tau^{X, e(h)}$, and $\tau_{\mathrm{s}}^{e}$ are the corresponding total spin lifetimes; $\omega_{\mathrm{L}}$ is the electron Larmor angular frequency; $\phi_{0}$ is the relative phase shift of the exciton precession, and $\phi$ is the IPS of the RESP precession. The total spin lifetime of the electron-in-exciton $\tau^{X, e}$ includes the recombination time $\tau_{\mathrm{r}}^{X}$, the time of exciton binding into a trion $\tau_{\mathrm{c}}$, and the spin relaxation time $\tau_{\mathrm{s}}^{X, e}$ (i.e. $1 / \tau^{X, e}=$ $\left.1 / \tau_{\mathrm{r}}^{X}+1 / \tau_{\mathrm{c}}+1 / \tau_{\mathrm{s}}^{X, e}\right)$. Since there is no change of the trion complexes to excitons, the excitons could be generated only at the very time of the photon excitation $(t=0)$. Therefore we can conclude that $\phi_{0}=0$. It is noted that the first, second, and third terms in Eq. 1 disappear rapidly due to their short lifetimes $(\sim 10 \mathrm{ps}, \sim 10 \mathrm{ps}$, and $<100 \mathrm{ps,} \mathrm{re-}$ spectively). The evolution of the first three terms is difficult to capture, while the fourth term remains over the range of a few ns. In the case of Fig. 2, the exciton component is negligible since the QW was excited selectively at the trion resonant transition. Thus, the TRKR signal can be fitted safely by the fourth term of Eq. 1 over the time range of $t=0.3-3 \mathrm{~ns}$. For the fitting curve, the extrapolating back to zero time delay $(t=0)$ can be seen as the time delay of RESP generation or the temporal shift $\phi / \omega_{\mathrm{L}}(=-19.6 \mathrm{ps})$ of the time origin as indicated in the inset (III) of Fig. 2. The temporal shift can be converted to IPS $=-0.47$ rad. Therefore, the initial phase $\phi$ of the RESP precession plays out a 

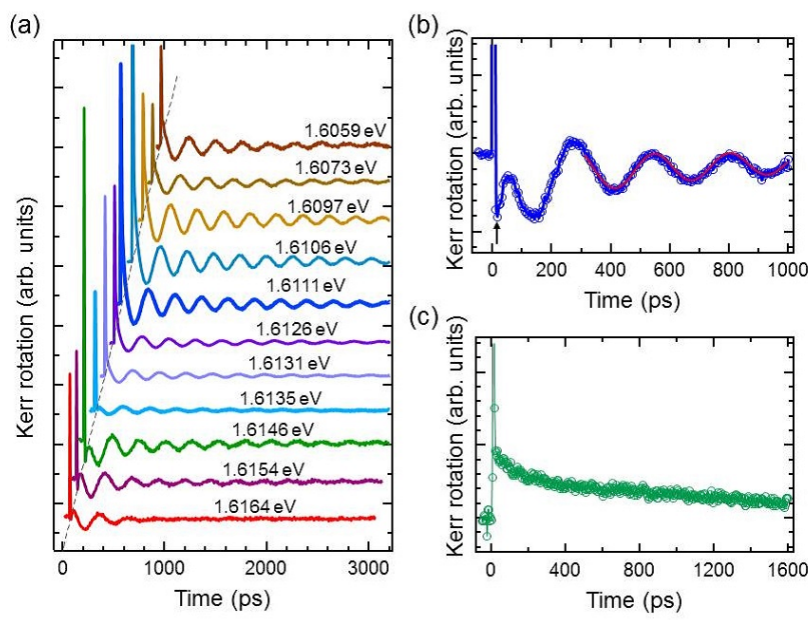

Figure 3 (Color Online) (a) TRKR signals for different excitation energy $(189 \mathrm{mT}, 10 \mathrm{~K})$. Two traces with the thick lines correspond approximately to the trion and exciton resonant conditions, respectively. (b) A typical TRKR signal (blue) and the fitting curve of the electron spin precession (red) at $1.6140 \mathrm{eV}(189 \mathrm{mT}, 10$ K). (c) A TRKR signal (green) at $1.6140 \mathrm{eV}(0 \mathrm{mT}, 10 \mathrm{~K})$.

slightly negative IPS according to this experimental result. The negative IPS is very interesting because it requires the asymmetric hole spin-flips, which was confirmed by some measurements such as the power dependence of an additional pump pulse and so on [10].

3.2 Excitation energy dependence of IPS In this report, we have performed the TRKR measurements when the excitation photon energy was scanned from 1.6059 $\mathrm{eV}$ (lower than the trion transition) to $1.6164 \mathrm{eV}$ (higher than the exciton transition). Some of the TRKR signals are shown in Fig. 3 (a). For an easy view, the time origin (dashed line) of each signal is shifted in the figure. According to these results, we can see the contribution features of the trions and excitons to IPS of RESP over a large range of the pumping photon energy. The maximum amplitude of the RESP signal was obtained at the trion resonance. Seen from the figure, some unusual features in the range of 0 200 ps have been observed around the exciton resonance, where there is an extremely fast downward decay and an upswing.

An example of the unusual TRKR signal measured with a pump power of $8 \mathrm{~mW}$ at $10 \mathrm{~K}$ in $B_{x}=189 \mathrm{mT}$ is displayed in Fig. 3 (b) (not shown in Fig. 3 (a)) and its fitting curve by the third and fourth terms of Eq. 1 is added in the range of $0.3-3 \mathrm{~ns}$. Here, the excitation energy was tuned to $1.6140 \mathrm{eV}$ which was a little higher than the exciton resonant transition energy. No directly-generated trion can be assumed due to the exciton resonant excitation with a low power excitation. For all the analyses below, the condition $2 \pi / \omega_{\mathrm{L}}>\tau_{\mathrm{r}(\mathrm{s}, \mathrm{c})}^{T(X)}$ is applicable. As shown in Fig. 3 (b), there is a clear fast drop close to the time origin (marked

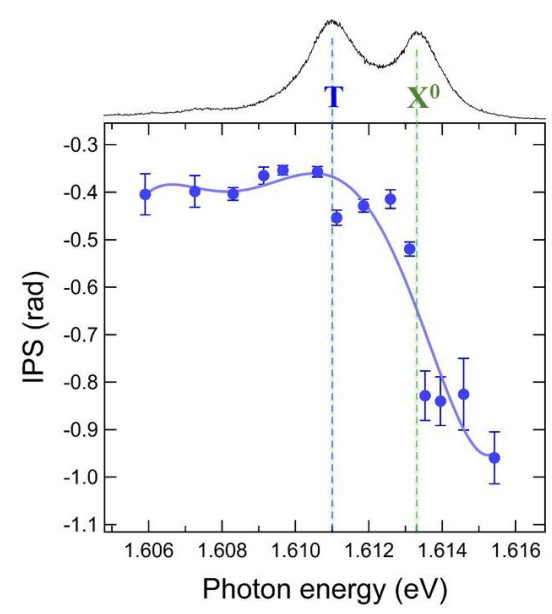

Figure 4 (Color Online) The excitation photon energy dependence of IPSs. The solid line is a guide for eyes. The blue and green dashed lines are used to mark the resonant transition energies of trion $(T)$ and neutral exciton $\left(X^{0}\right)$, respectively. The upper figure is the PL spectra at $10 \mathrm{~K}$.

by an arrow) in this typical signal. The excited initial signal $\left(X^{0}: \uparrow \Downarrow\right)$ by a $\sigma^{-}$pulsed excitation is defined as positive $\left(S_{\uparrow}=S_{\Downarrow}>0\right)$ at $t=0$ (thus, $S_{\downarrow}<0$ and $S_{\Uparrow}<0$ ). The signal goes down to below the balanced level largely from the initial spin polarization within tens of picoseconds. That is due to the spin precession of the electron-in-exciton (partly from $\uparrow$ to $\downarrow$ ) and a quick spin asymmetric relaxation of the heavy hole-in-exciton (from $\Downarrow$ to $\Uparrow$ ) which happens in trions as discussed in the former report [10]. After the point marked by an arrow, the TRKR signal rises again gradually due to the recombination of the transformed trions and excitons. Concretely speaking, the recombination of trions returns the electrons back to the resident electron ensemble to create additional RESP at later times. The subsequently created RESP induces a negative IPS and raises the spin polarization integrated throughout the entire trion lifetime.

To extract the time and relative amplitude of the shortliving precession component in the signal, the oscillatory part of this TRKR trace has been fitted. Then, the relative amplitude $A^{X, e}[1.6140 \mathrm{eV}](\sim 84.2)$ could correspond to the relative number of photo-generated excitons with a decay time $\tau^{X, e}[1.6140 \mathrm{eV}](\sim 57.6 \mathrm{ps})$. Under the same excitation condition, another TRKR signal obtained after removing the transverse magnetic field $B_{x}$ is shown in Fig. 3 (c). The total relative number of resident electrons after that all excitons decay to trions should be estimated as its fitted long-living RESP. Therefore, $A^{e}(1.6140 \mathrm{eV})(\sim 15.3)$ was obtained from the fitting by $S_{z}(t)=A^{X} \exp \left(-t / \tau^{X}\right)+$ $A^{e} \exp \left(-t / \tau_{\mathrm{s}}^{e}\right)$ for the range of 0.6-3 ns in Fig. 3(c). Then, the ratio between the exciton-to-trion transformation time $\tau_{\mathrm{c}}$ and the electron-in-exciton spin lifetime $\tau^{X, e}$ would be 
(a)

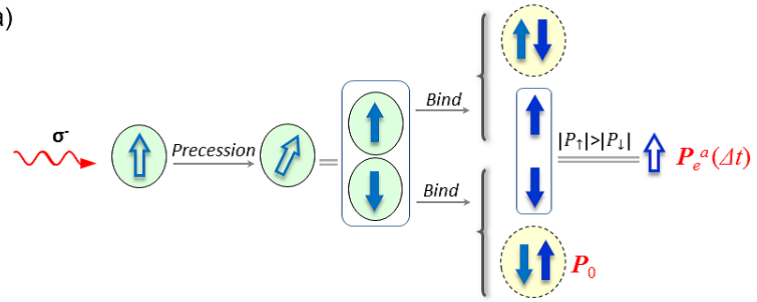

(b)

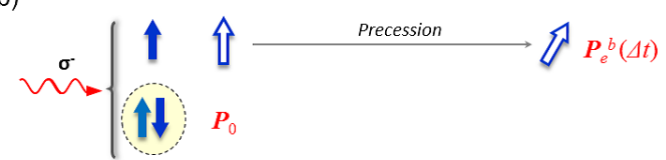

Figure 5 (Color Online) Simplified electron spin dynamics: (a) The first RESP formation from the photo-generated excitons; (b) The first RESP under trion resonant excitation. The electron-inexciton spin and the electron-in-trion spin are in the circles with the solid and dashed lines, respectively.

estimated to be [16]

$$
\tau_{\mathrm{c}} / \tau^{X, e}=A^{X, e} / 2 A^{e}=2.76 .
$$

Assuming that $\tau_{\mathrm{r}}^{X}, \tau_{\mathrm{c}} \ll \tau_{\mathrm{s}}^{X, e}$ and using $1 / \tau^{X, e}=$ $1 / \tau_{\mathrm{r}}^{X}+1 / \tau_{\mathrm{c}}+1 / \tau_{\mathrm{s}}^{X, e}$ with the above relation, we work out the exciton-to-trion transformation time $\tau_{\mathrm{c}} \sim 157 \mathrm{ps}$ and electron-in-exciton spin lifetime $\tau^{X, e} \sim 57 \mathrm{ps,} \mathrm{which}$ agrees well with the fitting parameter above $\left(\tau^{X, e} \sim 57.6\right.$ ps). In the past work, the direct transformation from excitons to trions has also been proved by the PL and PL excitation experiments [17].

Figure 4 summarizes the corresponding IPSs of RESP precession which are displayed by the solid circles through fitting the TRKR signals for the excitation energy in the range of $1.6059 \mathrm{eV} \sim 1.6164 \mathrm{eV}(772.2 \mathrm{~nm} \sim 767.1 \mathrm{~nm})$. As shown from the trend of IPSs indicated by a solid line, the negative IPS is almost constant $(\sim-0.40 \mathrm{rad})$ with varying the excitation energy from $1.6059 \mathrm{eV}$ to $1.6126 \mathrm{eV}$ around the trion resonance, in which case the trion spin dynamic is dominant. However, the negative IPS of RESP suddenly increases to $-0.83 \mathrm{rad}$ when the photon energy reaches 1.6135 $\mathrm{eV}$ just after passing over the neutral exciton resonance. Subsequently, the absolute value of the negative IPS continues to raise with the increasing excitation energy. After ruling out other possibilities, the excitons are supposed to be formed and then to decay to some negative trions as well as to create the later additional RESP.

Finally we summarize the RESP dynamics and the origin of the larger absolute IPS in the case of the exciton excitation. Figure 5 shows the different spin evolution between the electron-in-exciton spin (Fig. 5 (a)) and resident electron spin after trion generation (Fig. 5 (b)). For the simplicity, the heavy hole spin dynamics and the spin-flips of electron are skipped in this diagram. The electron-inexciton spin and the electron-in-trion spin are in the circles with the solid and dashed lines, respectively.

In the figure, we describe that the excitons and trions are excited at the same time origin $(t=0)$ corresponding to the exciton resonant and trion resonant excitations, respectively. In the case of the trion excitation (Fig. 5 (b)), the same amount of spin polarization $P_{0}$ for RESP and trions are created instantly after the excitation [10]. On the other hand, in the case of the photo-generated neutral excitons (Fig. 5 (a)), a certain time interval of $\Delta t\left(\ll \pi / 2 \omega_{\mathrm{L}}\right)$ is necessary in order to affect the RESP since the excitons have to capture resident electrons with a proper spin orientation and transform to trions with the same amount of $\left|P_{0}\right|$.

During the interval $\Delta t$, both the electron-in-exciton spin polarization and the RESP (the hollowed arrows in Fig. 5 (a) and (b), respectively) experience the precession by the phase of $\omega_{\mathrm{L}} \Delta t$ under the transverse magnetic field. Therefore, the spins of the electron-inall excitons would be composed of the $\uparrow$ - and the $\downarrow$ components. At the same time, the heavy hole-in-exciton spins have been asymmetrically flipped $(\Downarrow \rightarrow \Uparrow)$ as do trions in this QW [10]. Since the holes have no spin precession, they always induce the returned electron spin as a net spin-up or spin-down, never as the superposition. Then, the left unpaired resident electron spins from the above excitons binding to trions are made up of the $\uparrow$ part: $\cos \left(\omega_{\mathrm{L}} \Delta t / 2\right) P_{0}$ and the $\downarrow$-part: $\sin \left(\omega_{\mathrm{L}} \Delta t / 2\right) P_{0}$. In other words, the formed RESP is represented by $P_{e}^{a}(\Delta t)=\left\{\cos \left(\omega_{\mathrm{L}} \Delta t / 2\right)-\sin \left(\omega_{\mathrm{L}} \Delta t / 2\right)\right\} P_{0}$ and just starts to oscillate with the electron Larmor frequency all over again at $t=\Delta t$ along with the evolution form of $\left|\cos \left(\omega_{\mathrm{L}} \Delta t / 2\right)-\sin \left(\omega_{\mathrm{L}} \Delta t / 2\right)\right| P_{0} \cos \left\{\omega_{\mathrm{L}}(t-\Delta t)\right\}$. Obviously, $P_{e}^{a}(\Delta t)$ lets the phase shift back $\left(\omega_{\mathrm{L}} \Delta t\right)$ to zero at $t=\Delta t$ and is considered to induce an initial phase shift $-\omega_{\mathrm{L}} \Delta t$ at $t=0$. That is why the precession trace seems to start at another time off the real time origin, as seen in Fig. 3 (b). Nevertheless, we know the component RESP $P_{e}^{b}(\Delta t)$ immediately starts to precess after formation and proceeds smoothly in Fig. 5 (b). Thus $P_{e}^{b}(\Delta t)$ could not contribute any initial phase shift. In brief, $P_{e}^{a}(\Delta t)$ lags in phase, which is the main reason of a larger negative IPS for the exciton excitation.

4 Conclusion In summary, we systematically investigated the negative IPSs in the TRKR traces of RESP under a low magnetic field. According to these results, the significant role played by $e-h h$ pairs on the IPS has been indicated. Even there is no substantial change in the IPSs induced by trion spin dynamics under the low intensity excitation. By extending the excitation energy beyond the trion transition regime, the absolute value of negative IPS becomes larger and larger with increasing the excitation energy. This is because the newly formed RESP rewinds to the beginning after the transformation from $X^{0}$ to $T$. In ad- 
dition, the transformation time from $X^{0}$ to $T$ was deduced from the careful analysis of TRKR signals. More importantly, these clear effects of excitons and the dynamic physical picture of IPS are important for studying and controlling the electron spin dynamics in semiconductor nanostructures.

Acknowledgements This work was supported in part by JSPS KAKENHI (Grants No. 25247047 and No. 26800162). The research in Poland was supported by the National Science Centre (Poland) through Grant No. DEC-2014/14/M/ST3/00484 and by the Foundation for Polish Science through the Master Program.

\section{References}

[1] D. D. Awschalom, N. Samarth, and D. Loss, Semiconductor Spintronics and Quantum Computation (SpringerVerlag, Berlin, 2002).

[2] Spin Physics in Semiconductors, Springer Series in SolidState Sciences Vol. 157, Chaps. 2, 3 and 6, edited by M. I. Dyakonov (Springer-Verlag, Berline Heidelberg, 2008).

[3] M. M. Glazov, Phys. Solid State 54, 1 (2012).

[4] W. H. Lau and M. E. Flatte, J. Appl. Phys. 91, 8682 (2002).

[5] J. Tribollet, F. Bernardot, M. Menant, G. Karczewski, C. Testelin, and M. Chamarro, Phys. Rev. B 68, 235316 (2003).

[6] M. T. Portella-Oberli, J. Berney, L. Kappei, F. MorierGenoud, J. Szczytko, and B. Deveaud-Plédran, Phys. Rev. Lett. 102, 096402 (2009).

[7] H. Hoffmann, G. V. Astakhov, T. Kiessling, W. Ossau, G. Karczewski, T. Wojtowicz, J. Kossut, and L. W. Molenkamp, Phys. Rev. B 74, 073407 (2006).

[8] E. Vanelle, M. Paillard, X. Marie, T. Amand, P. Gilliot, D. Brinkmann, R. Levy, J. Cibert, and S. Tatarenko, Phys. Rev. B 62, 2696 (2000).

[9] J. M. Kikkawa, I. P. Smorchkova, N. Samarth, and D. D. Awschalom, Science 277, 1284 (1997).

[10] L.-P. Yan, M. Kurosawa, R. Kaji, G. Karczewski, S. Takeyama, and S. Adachi, Phys. Rev. B 90, 205307 (2014).

[11] L.-P. Yan, M. Kurosawa, W.-T. Hsu, W.-H Chang, and S. Adachi, Jpn. J. Appl. Phys. 54, 033003 (2015).

[12] G. V. Astakhov, V. P. Kochereshko, D. R. Yakovlev, W. Ossau, J Nürnberger, W. Faschinger, G. Landwehr, T. Wojtowicz, G Karczewski, and J. Kossut, Phys. Rev. B 65, 1153102002.

[13] G. V. Astakhov, M. M. Glazov, D. R. Yakovlev, E. A. Zhukov, W. Ossau, L. W. Molenkamp, and M. Bayer, Semicond. Sci. Technol. 23, 114001 (2008).

[14] S. Tomimoto, S. Nozawa, Y. Terai, S. Kuroda, K. Takita, and Y. Masumoto, Phys. Rev. B 81, 125313 (2010).

[15] M. T. Portella-Oberli, V. Ciulin, S. Haacke, J.-D. Ganiére, P.Kossacki, M.Kutrowski, T.Wojtowicz, andB.Deveaud, Phys. Rev. B 66, 155305 (2002).

[16] E. A. Zhukov, D. R. Yakovlev, M. Bayer, M. M. Glazov, E. L. Ivchenko, G. Karczewski, T. Wojtowicz, and J. Kossut, Phys. Rev. B 76, 205310 (2007).

[17] Z. Chen, R. Bratschitsch, S. G. Carter, S. T. Cundiff, D. R. Yakovlev, G. Karczewski, T. Wojtowicz, and J. Kossut, Phys. Rev. B 75, 115320 (2007). 